\title{
The epidemiology of trypanosomiasis in Rumphi district, Malawi: a ten year retrospective study Mwayiwawo Madanitsa ${ }^{1}$, John Chisi $^{2}$, Bagrey Ngwira ${ }^{3}$
}

1. College of Medicine, Malawi

2. Department of Haematology, College of Medicine, Malawi

3. Department of Community Health, College of Medicine, Malawi

Corresponding Author: Madanitsa Mwayiwawo, College of Medicine,

E.mail:mmadanitsa2004@yahoo.com or mmadanitsa@medcol.mw

\begin{abstract}
Background Human African Trypanosomiasis (HAT) is caused by two species of the tsetse fly vectored protozoan hemoflagellates belonging to Trypanosma brucei, namely T.b gambiense which predominates in Western Africa and follows a chronic disease course and T.b rhodensiense which is more prevalent in Southern and Eastern Africa, Malawi included, and follows a more acute and aggressive disease course. Previous studies in the Democratic Republic of Congo, Angola, Uganda and Sudan have demonstrated that the prevalence rates of $T . b$ rhodensiense infection have reached epidemic proportions.
\end{abstract}

Objectives'To describe the epidemiology of Trypanosomiasis in Rumphi District over the past ten years.

Methodology A total of 163 records from January 2000 to December 2006 were retrospectively studied.

Results There were more males than females (121 vs. 40) with the $20-29$ years age bracket having the highest number of cases $(26.3 \%, n=160)$. Stage 2 HAT was the commonest stage at presentation $(58.2 \%, \mathrm{n}=158)$ with the patients in the same being 3.5 times more likely to die than those with stage 1 HAT. Case fatality rates for late and early stage disease were $21.5 \%(\mathrm{n}=92)$ and $7.2 \%(\mathrm{n}=66)$ respectively with $84.6 \%$ having been cured $(n=162)$. Convulsions were associated with fatal disease outcome and the majority of cases $(97.2 \%$, $n=103$ ) lived within 5 kilometres of the Vwaza game reserve boundary.

Conclusion More men have been infected than women, with a high involvement in the $20-29$ age brackets. A dramatic increase with active case finding indicates a high under-detection of the disease with late stage HAT being predominant at presentation. Though it has been found that cases with late stage disease have an increased likelihood of dying compared to those in early stage HAT, the high proportion of successful treatment indicates that the disease still carries a high degree of favourable outcome with treatment. It has also been demonstrated in this study that more than $95 \%$ of trypanosomiasis cases live within $5 \mathrm{~km}$ of game reserve boundary. Disease interventions should be implemented in areas within $5 \mathrm{~km}$ of marshland game reserve boundary as priority areas.

\section{Introduction}

Human African Trypanosomiasis (HAT) or sleeping sickness is an important but equally neglected tropical disease in sub-Saharan Africa with an estimated 60 million people at risk in nearly 200 separate active foci from 36 sub-Saharan countries $^{1}$. The location of endemic foci of HAT follows the distribution of tsetse flies ${ }^{2}$. In Malawi, foci areas are in the districts of Rumphi, Nkhotakota, Ntchisi and Kasungu. The disease is caused by protozoan hemoflagellates belonging to
Trypanosoma brucei with two species causing distinct disease patterns in humans namely Trypanosma brucei gambiense and Trypanosma brucei rbodensiense ${ }^{1}$. T.b gambiense is found in central, west and some parts of eastern Africa, whereas T.b rhodesiense is found in southern and eastern Africa ${ }^{1}$. T.b rhodensiense infection is usually acute, causing severe symptoms and death within a few days or weeks. However, studies in Malawi have shown T.b rhodensiense with a chronic disease profile ${ }^{3}$. T.b gambiense infections tend to progress more slowly (over several years) and causes less severe symptoms ${ }^{1}$.

Human infection occurs sporadically in individuals who come into contact with the zoonotic cycle such as poachers, hunters, firewood collectors and tourists ${ }^{4}$ Inoculation of the protozoa by the vector (Glossina sp) into the blood stream is followed by an initial stage of proliferation at the site of the bite, causing local inflammation (chancre) with subsequent invasion of the hemolymphatic system marking what is known as the early stage HAT, also known as stage 1 HAT. Concomitant involvement of the central nervous system leads to meningocephalitic stage of the disease, also referred to as late stage or stage 2 HAT. Without treatment, the disease progresses to coma and death ${ }^{3}$. Stage 1 is treated with suramin, but stage 2 can only be treated with melarsoprol, a toxic arsenic derivative that is associated with the development of a severe post-treatment reactive encephalopathy (PTRE) ${ }^{5}$. This severe adverse reaction can occur in up to $10 \%$ of cases ${ }^{6,7}$, and may be fatal in $50 \%$ of the cases ${ }^{8}$.

HAT has been described as a major public health problem in sub-Saharan Africa, where it affects mainly the rural poor populations. The most recent prevalence estimates from the World Health Organization are 50,000-70,000 cases, based on a total number of 17,500 new cases reported per year in Africa?' The disease has been reported to have reached epidemic proportions in four countries, with a prevalence of over 20\% in some areas of: Sudan, Uganda, the Democratic Republic of Congo, and Angola ${ }^{4,10,11,12}$. It is well appreciated that cases tend to cluster in close proximity to animal reservoirs such as game parks. Studies on the effect of distance to health care in relation to stage of presentation have previously been carried out and have shown that the closer the patient lives to the reporting health facility, the greater the likelihood to that they will be detected with the early stage of infection ${ }^{13}$. It has also been demonstrated that the prognosis of late stage disease is poorer than in early stage disease ${ }^{14}$.

WHO estimates that current HAT control activities reach only $10 \%$ of persons at risk $^{15}$. With the results from previous studies in other countries suggesting resurgences and epidemics of HAT, it is important to describe the local epidemiology of HAT in order to estimate the burden of disease attributable to HAT in Malawi and to target interventions for the communities at risk. This study was therefore designed to describe the epidemiology of HAT in Rumphi District which is one of the recognized Trypanosomiasis rhodensiense endemic areas in Malawi. 


\section{Methods}

This was a retrospective study which reviewed hospital records from the laboratory and registry offices, of Trypanosomiasis cases from January 1997 to December 2006 at Rumphi District Hospital, which is located in the northern Region of Malawi, where Vwaza game reserve and Nyika National Parks are located. The populaces of the district are mainly subsistence farmers and tobacco growing is a major agricultural activity.

Clinical records of all confirmed cases of Trypanosomiasis that were recorded at the district hospital were retrieved for analysis. In all the cases, blood and cerebrospinal fluid (CSF) samples were taken for diagnosing and staging of Trypanosomiasisinfection. The diagnosis of Trypanosomiasis infection was confirmed by demonstration of parasites in the blood or CSF. The presence of parasites in CSF denoted stage 2 and presence of parasites in the blood only denoted stage 1 infection.

\section{Data Collection}

Data on patient age, gender, village of residence, stage of disease at presentation and disease outcome was extracted from clinical records and double entered into a Microsoft Access database. Geographical coordinates for villages of residence for all the cases were collected using a portable Global Positioning System (Garmin etrex, Garmin International, Inc USA (C) 2004 Garmin Ltd).

\section{Data Analysis}

Analysis was done using Intercooled STATA version 9.0 (StataCorp LP, 4905 Lakeway Drive, College Station, TX 77845 USA) and maps to depict spatial characteristics of interest in the study were created in ArcView GIS Version 3.1 (ArcView GIS Version 3.1 C 1992-1998, Environmental Systems Research Institute, Inc). Graphs were produced using Microsoft Excel software.
The associations between signs and symptoms with stage of presentation and disease outcome were tested using the Pearson Chi-Square test or the Fisher's Exact test were appropriate at the $5 \%$ level of significance. In order to examine the relationship of spatial distribution of cases and disease stage at presentation with respect to distance from the treating unit, the Mantel-Haenszel $\chi^{2}$ test was used. Logistic regression was used to assess the factors associated with the odds of dying from Trypanosomiasis.

\section{Ethical Consideration}

Ethical approval and oversight was granted by the College of Medicine Research and Ethics Committee (COMREC).

\section{Results}

\section{Demographic characteristics of Trypanosomiasis cases}

In this study, 163 records of confirmed Trypanosomiasis cases occurring over a period of 7 years were available. The age and sex distribution of the cases is shown in Figure 1. Of note, there were more males than females (3:1). The overall median age was 25 years (range $0-70$ years) with adults accounting for $67.3 \%$ of the cases.

\section{Temporal trends in the number of Trypanosomiasis cases}

The trends in the number of confirmed cases, being attended to by the hospital, showed a general rise over the 7 year period under study, with a marked increase in 2003 (Figure 2).

\section{Clinical characteristics of Trypanosomiasis cases}

There was a predominance of Stage 2 HAT cases at presentation, accounting for $58.2 \%$ of the cases. Treatment outcomes were considerably good despite the fact that most of the cases presented in the late stage of disease. In this cohort of patients, mortality was $15.4 \%$ with $80 \%$ of the

Figure 1: Age and sex distribution of HAT cases by sex at Rumphi District Hospital (2000 - 2006)

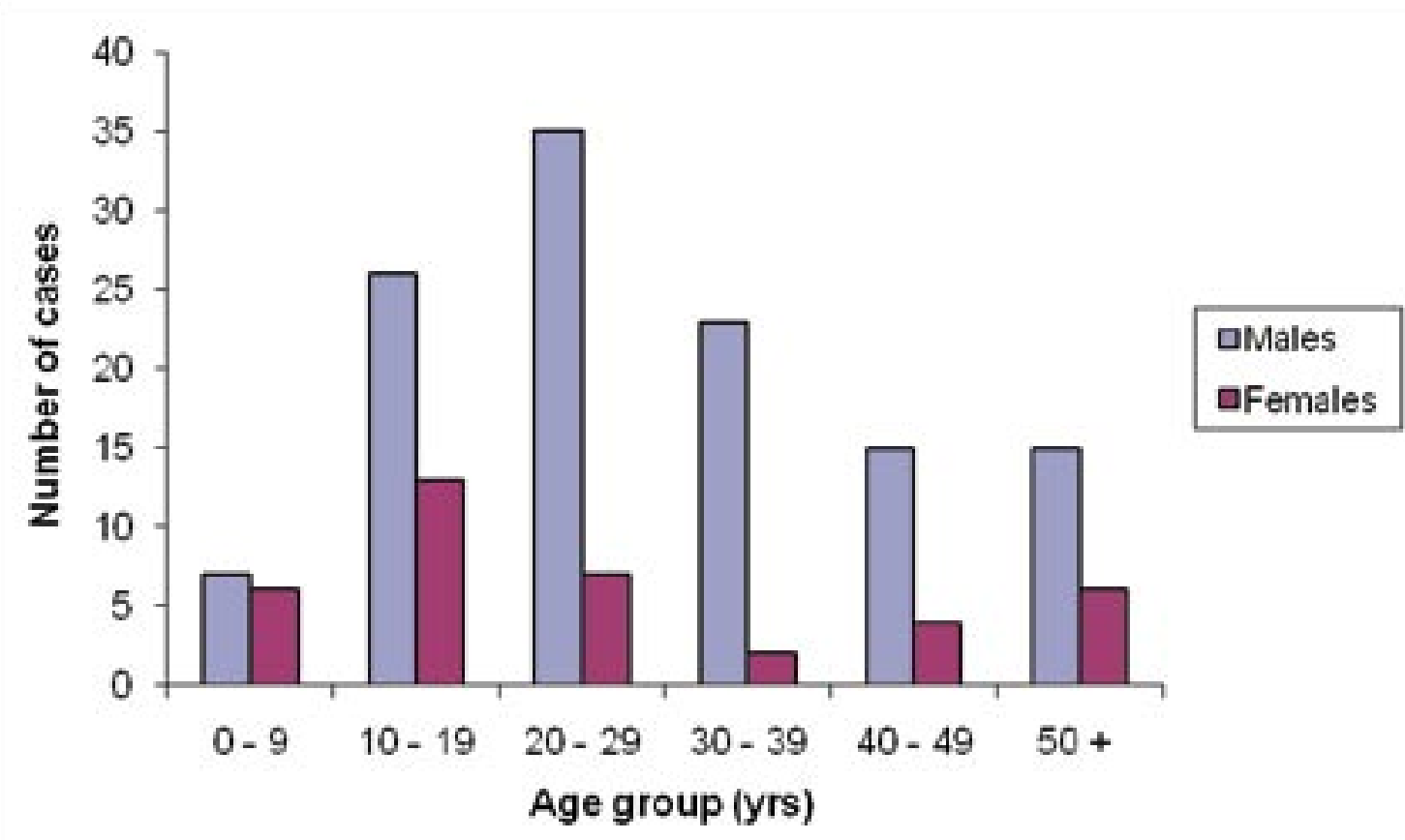




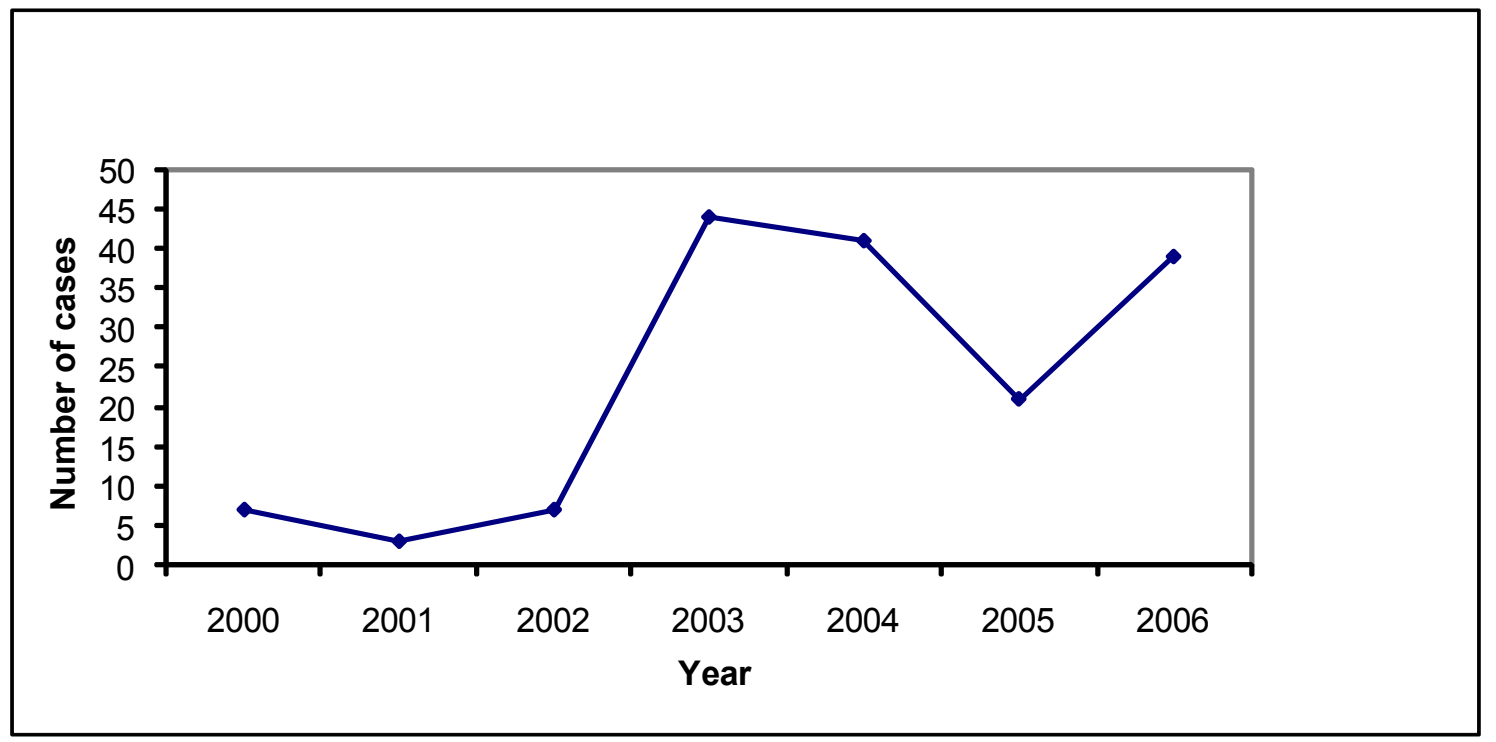

Figure 3: Distance of villages of residence of trypanosomiasis cases from game reserve boundary (2000 - 2006)

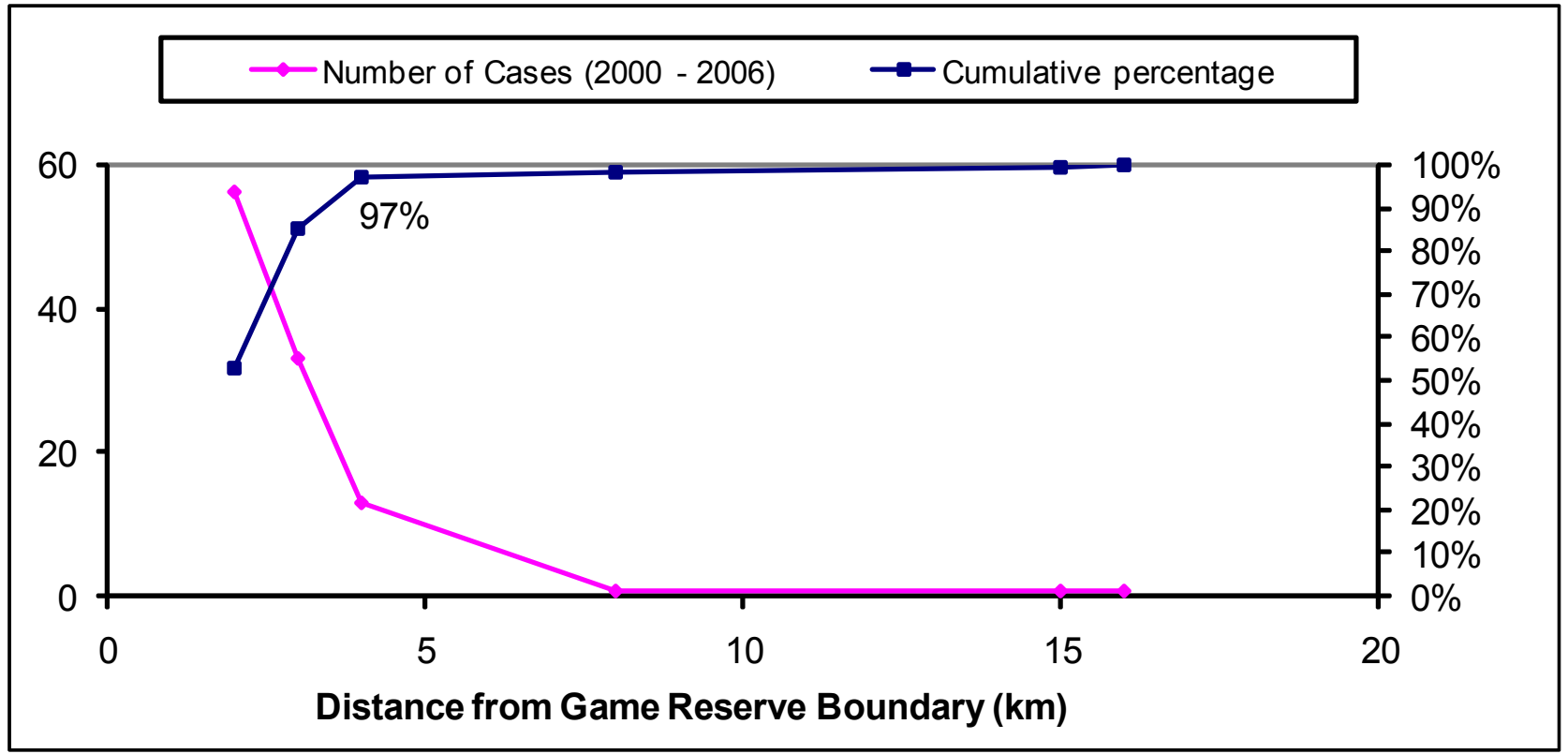

deaths having had late stage disease. Case fatality rates for late and early stage disease were $21.5 \%(\mathrm{n}=92)$ and $7.2 \%$ $(n=66)$ respectively with $84.6 \%$ having been cured $(n=162$. Fever was the most reported symptom in both stages (Early stage $=50 \%$; Late stage $=40.7 \%$ ). However, there was no statistically significant difference in the proportion of patients with fever as a symptom between the two clinical stages and no associations were found between any of the remaining documented symptoms or signs with stage at presentation (Table 1).

\section{Factors associated with outcome}

Treatment outcome was significantly associated with stage at presentation $(\mathrm{p}=0.01)$ and presence of convulsions $(p=0.005)$. Multivaraite analyses indicated that after adjusting for the presence of convulsions, individuals who presented with Stage 2 HAT were more likely to die than those with Stage 1 HAT (odds ratio $(\mathrm{OR})=3.51$, 95\% Confidence Interval (CI) 1.24 - 9.88). Fever was not associated with either outcome (45.5\% amongst those that survived, 50\% in those that died, (Table 2).

\section{Spatial distribution of Trypanosomiasis cases}

Most of the cases, $97.2 \%(\mathrm{n}=103)$ came from within $5 \mathrm{~km}$ of the game reserve boundary (Figure 3). The vast majority of cases were clustered along the Vwaza Game Reserve boundary compared to Nyika Game Reserve as demonstrated by Geo-referencing (Figure 4). We did not find an association between distance from diagnostic facility and stage at presentation $(\mathrm{p}$-value $=0.60)$ or outcome $(\mathrm{p}$ value $=0.32$.

\section{Discussion}

This data has shown that more men are infected with Trypanosomiasis in Rumphi district. This could be attributed to greater exposure to the parasite by men because of the nature of the activities which they engage in which include poaching, honey gathering and encroachment into the game reserve to clear land for tobacco farming, which is a major economic activity in this area. It is within the same vein that the greater proportions of cases are adults owing to activities that bring them into contact with the infected vectors.

We also found that in general there was an increase in the 


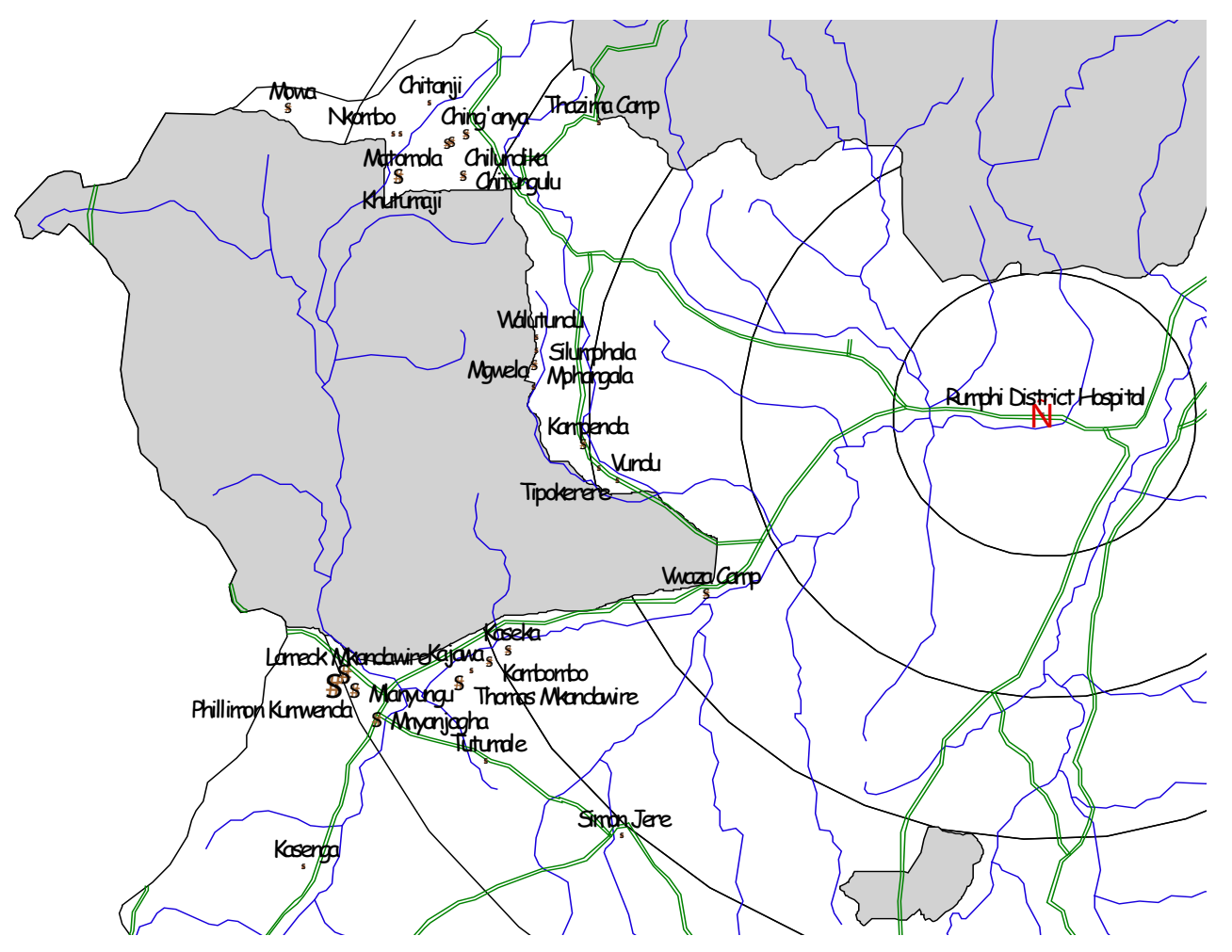

number of cases of Trypanosomiasis over the 7 year period understudy. However, there was a marked increase that was noted in 2003 by $600 \%$. This could be attributed to active case finding that was done that year with support from the East African Network for Trypanosomiasis (EANET) as part of a Trypanosomiasis surveillance programme. Such a change concurs well with findings from a previous study conducted in Uganda of under-detection of T.b. rhodensiense infection ${ }^{16}$.

With more than $50 \%$ of the cases having been diagnosed with late stage disease, the study findings run in tandem with findings of a study conducted in $\mathrm{Uganda}^{14}$ in which approximately half the Trypanosomiasis patients presented with late stage disease. This finding could be due to weak surveillance. It could also be attributed to possible causative factors described as either patient factors or the health system. Some of these factors could include poverty of endemic countries, poverty of patients and low sensitivity of the diagnostic techniques ${ }^{13}$. In Rumphi, distance from the health facility does not seem to influence stage at presentation. As such, other parameters could be responsible for this observation, such as patient perceptions toward the seriousness of the disease, quality of health care or access to health education. These parameters were not assessed in the current study.

There was a greater chance of dying with late stage disease than early stage. This observation is in agreement with observations from a previous study that showed that the prognosis of late stage sleeping sickness presentation is significantly poorer that in the one in the early stage of the disease $\mathrm{e}^{14}$. There were marked successful outcomes in treatment. These outcomes are likely due to the efficacy of the treatment and management at the hospital. Fever was the most reported symptom in both stage 1 and 2 HAT. However, there was no statistically significant association to warrant it as a clinical predictor of disease stage. There was a significant association between convulsions and fatal outcome. As such, convulsions could serve as a prognostic indicator of HAT.

The vast majority of cases were in close proximity to Vwaza game reserve which is a marsh land compared to Nyika game reserve which is mountain ecology. Such spatial distribution of the cases agrees with observations in a study done in $2005^{16}$ where proximity to swampland was predictive of $T . b$ rhodensiense infection. This observation could be attributed to an ecology dependant factor for the reservoir vector which is favoured by marsh land. This however, was not assessed in the current study.

The greatest limitation encountered in the study was the unavailability of records dating from 1997 to 1999. Other limitations included the poor recording of clinical parameters with lack of specific definitions for signs and symptoms and the inability to trace the areas of residence of some of the cases. Despite the limitations, this study managed to attain a satisfactory number of cases as well as having clear definitions for disease stage at presentation. Demographic and clinical outcome data was clearly documented from the available records. Additionally, this is the first retrospective study that has described in detail the epidemiology of Trypanosomiasis in a recognized endemic area in Malawi.

\section{Conclusion}

This study indicates that over the past seven years, more men have been infected than women, with a high involvement in the $20-29$ age brackets. A dramatic increase with active case 
Table 1: Relationship between symptoms and signs with stage at presentation

\begin{tabular}{|c|c|c|c|}
\hline Symptoms and Signs & $\begin{array}{l}\text { Early } \\
\text { (frequency) }\end{array}$ & $\begin{array}{l}\text { Late } \\
\text { (frequency) }\end{array}$ & $\begin{array}{l}\text { p value } \\
\text { (Fisher's } \\
\text { exact test) }\end{array}$ \\
\hline Dizziness & 3 & 3 & 0.68 \\
\hline Convulsions & 1 & 2 & 0.56 \\
\hline Headache & 15 & 8 & 0.60 \\
\hline Palpitations & 4 & 0 & 0.14 \\
\hline Poor vision & 3 & 1 & 0.64 \\
\hline Abnormal behaviour & 2 & 2 & 1.00 \\
\hline Vomiting & 4 & 0 & 0.14 \\
\hline Abdominal pain & 6 & 5 & 0.75 \\
\hline General body pains & 13 & 8 & 1.00 \\
\hline $\begin{array}{l}\text { General body } \\
\text { weakness }\end{array}$ & 8 & 6 & 1.00 \\
\hline Yellow eyes & 0 & 2 & 0.16 \\
\hline Abnormally sleepy & 8 & 7 & 0.57 \\
\hline Oral sores & 2 & 1 & 1.00 \\
\hline Speech deficit & 1 & 4 & 0.15 \\
\hline Low consciousness & 3 & 3 & 0.68 \\
\hline Cough & 5 & 2 & 0.69 \\
\hline Chest pains & 4 & 3 & 1.00 \\
\hline Fever & 20 & 11 & 0.62 \\
\hline Weight loss & 4 & 2 & 1.00 \\
\hline Swollen face & 1 & 0 & 1.00 \\
\hline Anaemia & 4 & 6 & 0.29 \\
\hline Lymphadenopathy & 6 & 4 & 1.00 \\
\hline Hepatomegaly & 2 & 1 & 1.00 \\
\hline Splenomegaly & 2 & 4 & 0.21 \\
\hline Edema & 3 & 5 & 0.25 \\
\hline Neck stiffness & 3 & 3 & 0.68 \\
\hline Tachycardia & 1 & 0 & 1.00 \\
\hline Jaundice & 0 & 2 & 0.16 \\
\hline
\end{tabular}

finding indicates a high under-detection of the disease with late stage HAT being predominant at presentation. Though it has been found that cases with late stage disease have an increased likelihood of dying compared to those in early stage HAT, the high proportion of successful treatment indicates that the disease still carries a high degree of favourable outcome with treatment. It has also been demonstrated in this study that more than $95 \%$ of trypanosomiasis cases live within $5 \mathrm{~km}$ of game reserve boundary.

In light of the findings in this study, it is recommended that disease interventions should be preferentially targeted to communities who live within $5 \mathrm{~km}$ of marsh land game reserve boundary. There is also a need to implement interventions that would lead to earlier presentation to the health facility in order to reduce the marked proportion of late stage presentations which are associated with more than a threefold chance of dying. Such interventions could include increased community sensitization campaigns and stronger disease surveillance.
Table 2: Relationship between symptoms and signs with outcome

\begin{tabular}{|c|c|c|c|}
\hline Symptoms and Signs & $\begin{array}{l}\text { Cured } \\
\text { (frequency) }\end{array}$ & $\begin{array}{l}\text { Died } \\
\text { (frequency) }\end{array}$ & $\begin{array}{l}\text { p value } \\
\text { (Fisher's } \\
\text { exact test) }\end{array}$ \\
\hline Dizziness & 3 & 3 & 0.07 \\
\hline Convulsions & 0 & 3 & 0.01 \\
\hline Headache & 21 & 2 & 0.20 \\
\hline Palpitations & 4 & 0 & 1.00 \\
\hline Poor vision & 3 & 1 & 0.56 \\
\hline Abnormal behaviour & 3 & 1 & 0.56 \\
\hline Vomiting & 3 & 1 & 0.56 \\
\hline Abdominal pain & 11 & 0 & 0.19 \\
\hline General body pains & 19 & 2 & 0.31 \\
\hline $\begin{array}{l}\text { General body } \\
\text { weakness }\end{array}$ & 10 & 4 & 0.26 \\
\hline Yellow eyes & 1 & 1 & 0.33 \\
\hline Abnormally sleepy & 10 & 5 & 0.12 \\
\hline Oral sores & 2 & 1 & 0.45 \\
\hline Speech deficit & 4 & 1 & 1.00 \\
\hline Low consciousness & 4 & 2 & 0.29 \\
\hline Cough & 6 & 1 & 1.00 \\
\hline Chest pains & 5 & 2 & 0.60 \\
\hline Fever & 25 & 6 & 1.00 \\
\hline Weight loss & 6 & 0 & 0.58 \\
\hline Swollen face & 1 & 0 & 1.00 \\
\hline Anaemia & 8 & 2 & 1.00 \\
\hline Lymphadenopathy & 10 & 0 & 0.19 \\
\hline Hepatomegaly & 3 & 0 & 1.00 \\
\hline Splenomegaly & 6 & 0 & 0.58 \\
\hline Edema & 5 & 3 & 0.15 \\
\hline Neck stiffness & 4 & 2 & 0.29 \\
\hline Tachycardia & 1 & 0 & 1.00 \\
\hline Jaundice & 2 & 0 & 1.00 \\
\hline
\end{tabular}

\section{Acknowledgements}

Acknowledgements with gratitude go to the following: EANET for funding, Professor John Chisi and Dr Bagrey Ngwira for guidance and mentoring throughout the course of the study, Dr Linda Kalilani-Phiri for supervision through the course of the study and the writing of the manuscript for publication, Dr Anna Molesworth for assistance rendered with geographic information systems, Mr. A Kumitawa, for statistical analysis, Mr Chirongo of Rumphi District Hospital for his assistance in the field work, the DHO and all members of staff of Rumphi District Hospital.

\section{References}

1. World Health Organization. Control and surveillance of African trypanosomiasis: report of a WHO expert committee. World Health Organization, Geneva; 1998.

2. Barrett, MP, Burchmore RJ, Stich A., Lazzari JO, Frasch AC, Cazzulo JJ, Krishna S. The trypanosomiases. Lancet 2003.;362:1469-1480.

3. MacLean L, Chisi JE, Odiit M, Gibson W, Ferris V, Picozzi K, Sternberg1 J M. Severity of human African trypanosomiasis in East Africa is associated with geographic location, parasite genotype, and host inflammatory cytokine response profile. Infect Immun 2004:7040-7044 
4. Smith DH, Pepin J, Stich AHR. Human African trypanosomiasis: an emerging public health crisis. Brit. Med. Bull. 1998;54:341-355

5. de Atouguia JLM, Kennedy PGE. Neurological aspects of human African trypanosomiasis. In: Davies LE, Kennedy PGE, editors. Infectious diseases of the nervous system. Oxford: ButterworthHeinemann, 2000; p. 321-72

6. Adams JH, Haller L, Boa FY, Doua F, Dago A, Konian K. Human African trypanosomiasis (T.b gambiense): a study of 16 fatal cases of sleeping sickness with some observations on acute reactive arsenical encephalopathy. Neuropathol Appl Neurobiol 1986; 12:81-94

7. Pepin J, Milord F. African trypanosomiasis and drug-induced encephalopathy: risk factors and pathogenesis. Trans R Soc Trop Med Hyg 1991;85:222-224

8. Pepin, J., Milord, F. The treatment of human African trypanosomiasis. Adv Parasitol. 1994;33:1-47

9. World Health Organization. Human African trypanosomiasis (sleeping sickness): epidemiological update. Wkly Epidemiol Rec. 2006;81:7180

10. Moore A., Richer M. Re-emergence of epidemic sleeping sickness in southern Sudan. Trop. Med. Int. Health 2001;6:342-347
11. Stanghellini A, Josenando T. The situation of sleeping sickness in Angola: a calamity. Trop. Med. Int. Health 2001;6:330-334

12. Van Nieuwenhove S., Betu-Ku-Mesu V.K., Diabakana P.M., Declercq J., Miaka C. Sleeping sickness resurgence in the DRC: the past decade. Trop. Med. Int. Health 2001;6: 335-341

13. Odiit M, Coleman PG, McDermott JJ, Fevre EM, Welburn SC and Woolhouse MEJ. Spatial and temporal factors for the early detection of Trypanosoma brucei rhodesiense sleeping sickness in Tororo and Busia districts, Uganda. Trans R Soc Trop Med Hyg 2004;98:569-576.

14. Odiit M, Kansiime F, Enyaru JCK. Duration of symptoms and case fatality of sleeping sickness caused by Trypanosoma brucei rhodesiense sleeping sickness in Tororo, Uganda. East Afr. Med J. 1997;74:792 $-795$

15. Lutumba P, Robays J, Miaka C, Kande V, Molisho D, Declercq J, et al. Trypanosomiasis control, Democratic Republic of Congo, 1993-2003. Emerg Infect Dis. 2005;11:1382-1388.

16. Odiit M, Coleman PG, Liu WC, McDermott JJ, Fe`vre EM, Welburn SC and Woolhouse MEJ. Quantifying the level of under-detection of Trypanosoma brucei rhodesiense sleeping sickness cases. Trop Med Int Health 2005;10(9):840-849.

\section{Essay Competition - Young Voices in Research for Health}

For the fourth time, the Global Forum and The Lancet invite young researchers and leaders of tomorrow to submit an essay to the popular competition "Young Voices in Research for Health": Innovating for the health of all.

The deadline for receipt of entries is 3 May 2009.

\section{Who can participate?}

If you are a young professional (under 30) working on or interested in the broad spectrum of research for health, please feel free to submit your original, provocative, idealistic and passionate ideas on this year's theme, taking established practices to task in a constructive fashion. You will find the participation guidelines here: http://www.globalforumhealth.org/shlinks/yvhr2009.php

\section{What is the theme?}

This year's competition focuses on "Innovating for the health of all". "Innovation" encompasses the entire process from the generation of new ideas, to their transformation into something useful, to their implementation. Innovation for health includes the development of new and more cost-effective services, products, methods, management practices and policies to improve health outcomes. It involves both social and technological innovation.

\section{What are the prizes?}

The 40 most outstanding essays will be published in an anthology and the authors are featured one by one on the Global Forum's home page throughout the year. From these, five or six winners from different geographical regions will be invited to Forum 2009, with all expenses paid (Havana, Cuba, from 16 to 20 November). http://www.globalforumhealth.org/shlinks/f2009.php

\section{Deadline: 3 May 2009}

You can submit your essay here: http://www.globalforumhealth.org/shlinks/yvhr2009.php (in English, French or Spanish) and/or help spread the word by forwarding this message. 